\title{
Analytical Validation and Performance of a 7-Gene Next-Generation Sequencing Panel in Uveal Melanoma
}

\author{
Katherina M. Alsina ${ }^{a} \quad$ Lauren M. Sholla Kyle R. Covington $^{a}$ \\ Suzzette M. Arnal ${ }^{a}$ Michael A. Durante ${ }^{b}$ Christina L. Decatur ${ }^{b}$ John F. Stone ${ }^{a}$ \\ Kristen M. Oelschlager ${ }^{\mathrm{a}} \quad$ J. William Harbour ${ }^{\mathrm{b}}$ Federico A. Monzon ${ }^{\mathrm{a}}$ \\ Robert W. Cook ${ }^{a}$ Sherri Borman ${ }^{a}$ \\ ${ }^{a}$ Castle Biosciences, Inc., Friendswood, TX (Headquarters) and (Laboratory), Phoenix, AZ, USA; \\ ${ }^{b}$ Bascom Palmer Eye Institute, University of Miami Miller School of Medicine, Miami, FL, USA
}

\section{Keywords}

Uveal melanoma · DNA sequencing · Gene expression profiling

\begin{abstract}
Introduction: Gene expression profiling (GEP) is widely used for prognostication in patients with uveal melanoma (UM). Because biopsy tissue is limited, it is critical to obtain as much genomic information as possible from each sample. Combined application of both GEP and next-generation sequencing (NGS) allows for analysis of RNA and DNA from a single biopsy sample, offers additional prognostic information, and can potentially inform therapy selection. This study evaluated the analytical performance of a targeted custom NGS panel for mutational profiling of 7 genes commonly mutated in UM. Methods: One hundred five primary UM tumors were analyzed, including 37 formalin-fixed paraffinembedded (FFPE) and 68 fine-needle aspiration biopsy specimens. Sequencing was performed on the lon GeneStudio S5
\end{abstract}

karger@karger.com www.karger.com/oop

Karger $\stackrel{\text { ' }}{5}$

GOPEN ACCESS
(C) 2021 The Author(s)

Published by S. Karger AG, Basel

This article is licensed under the Creative Commons AttributionNonCommercial-NoDerivatives 4.0 International License (CC BYNC-ND) (http://www.karger.com/Services/OpenAccessLicense) Usage and distribution for commercial purposes as well as any distribution of modified material requires written permission. platform to an average read depth of $>500 \mathrm{X}$ per region of interest. Results: The 7-gene panel achieved a positive percent agreement of $100 \%$ for detection of both single-nucleotide variants and insertions/deletions, with a technical positive predictive value of $98.8 \%$ and $100 \%$, respectively. Intraassay and inter-assay concordance studies confirmed the assay's reproducibility and repeatability. Discussion/Conclusion: The 7-gene panel is a robust, highly accurate NGS test that can be successfully performed, along with GEP, from a single small-gauge needle biopsy sample or FFPE specimen.

(c) 2021 The Author(s)

Published by S. Karger AG, Basel

\section{Introduction}

Uveal melanoma (UM) is a rare but deadly intraocular cancer, with up to $50 \%$ of patients developing distant metastatic disease [1]. A 15-gene expression profiling (GEP) test commercially available as DecisionDx-UM 
(Castle Biosciences, Inc., Friendswood, TX, USA) is routinely used in the USA and parts of Canada for prognostication of metastatic risk in UM patients [2-4] and is included as standard of care in national guidelines $[5,6]$. Testing is most commonly performed on a small tissue sample taken by fine-needle aspiration biopsy (FNAB) but can also be performed on formalin-fixed paraffinembedded (FFPE) tissue taken from enucleations. Since the vast majority of UM patients are treated with radiotherapy rather than enucleation and repeated or aggressive biopsies can increase the risk of complications, it is critical that a molecular prognostic testing platform provide as much individualized genomic information as possible from a single biopsy sample. The ability to perform both GEP testing and mutational profiling via highthroughput next-generation sequencing (NGS) from the same UM biopsy sample reduces complications related to multiple biopsies and has the potential to aid in diagnostic classification, determine eligibility for targeted therapies, and provide additional individualized prognostic information for patients.

Nearly all UMs harbor mutually exclusive mutations in either GNAQ, GNA11, CYSLTR2, or PLCB4, resulting in constitutive activation of the G-protein-receptor signaling pathway [7-11]. While mutations in these $4 \mathrm{Gq}$ signaling pathway genes are not correlated with UM prognosis, they appear to be required for tumor initiation and may be helpful in confirming the presence of a uveal melanocytic lesion [7, 10-14]. During subsequent tumor evolution, additional mutations are gained in either $E I$ $F 1 A X, S F 3 B 1$, or BAP1 [10] and have been associated with prognosis in UM patients. For instance, mutations in $E I$ $F 1 A X$ and SF3B1 are associated with relatively low and intermediate metastatic risk (Class 1 GEP), respectively, while inactivating mutations in $B A P 1$, a tumor suppressor gene, are associated with high metastatic risk (Class 2 GEP) $[7,14,15]$.

A 7-gene sequencing panel, commercially available as DecisionDx-UMSeq (Castle Biosciences, Inc., Friendswood, TX, USA), utilizes NGS to identify variants within GNAQ, GNA11, CYSLTR2, PLCB4, EIF1AX, SF3B1, and $B A P 1$ using DNA isolated from primary UM tumor specimens. This study describes the development, analytical validation, and performance of this 7-gene panel. Performance characteristics for the target genes of interest were assessed according to the guidelines for validation of NGS-based oncology panels established by the Association for Molecular Pathology (AMP) and College of American Pathologists (CAP).

Validation of a 7-Gene Sequencing Panel for Uveal Melanoma

\section{Materials and Methods}

Tumor Specimens and Sample Preparation

The majority of primary UM tumor samples used in this study were acquired following routine clinical GEP testing at a centralized CAP-accredited, CLIA-certified, New York State Approved laboratory (Castle Biosciences, Inc., Phoenix, AZ, USA). During FNAB sample processing, an aliquot of the sample was set aside for clinical testing with the GEP test, and the remaining sample was frozen. These residual frozen samples, which would have otherwise been discarded after successful GEP testing, were de-identified, and $70 \mu \mathrm{L}$ were transferred to the clinical research scientists for analytical/technical validation of the 7-gene NGS panel. None of the research personnel were given access to identifiable patient health information, and results were not provided back to the patient or practitioner due to the strictly analytical nature of the study. Because no additional medical or privacy risks were incurred for these patients, patient consent was not required as described in published FDA guidance documents [16]. Institutional Review Board approval was also not required for the existing residual clinical specimens at Castle Biosciences because this analysis is exempt from the regulatory review requirements as set forth in section 46.101 (b) of 45 CFR 46. The subset of de-identified samples provided by the Harbour Lab was obtained with written patient informed consent and approval of the Institutional Review Board of the University of Miami (IRB-approved protocol number 20120773). For FFPE specimens derived from enucleations, samples were prepared as 5 -micron sections mounted onto 5 slides, and DNA extraction was performed with tumor cell content $\geq 80 \%$ following evaluation by trained pathologists.

\section{Nucleic Acid Extraction and Quantification}

Genomic DNA was extracted from FNAB samples using the AllPrep DNA/RNA Mini Kit (Qiagen, Hilden, Germany) and from FFPE samples using QIAamp DSP DNA Mini Kit (Qiagen, Hilden, Germany). DNA concentrations were determined by fluorometric quantitation using Qubit 2.0 Fluorimeter (Life Technologies, Waltham, MA, USA).

\section{Next-Generation Sequencing}

Libraries were prepared for each sample using a custom Ion Ampliseq Panel (Thermo Fisher Scientific, Waltham, MA, USA) following the manufacturer's instructions. A total of $15 \mathrm{ng}$ input DNA per sample (minimum $1.5 \mathrm{ng} / \mu \mathrm{L}$ sample DNA concentration) was loaded. Template preparation was performed on the Ion Chef System (Thermo Fisher Scientific, Waltham, MA, USA) using the Ion 510, Ion 520, and Ion 530 kit-chef (Thermo Fisher Scientific, Waltham, MA, USA). Samples were loaded onto an Ion 530 Chip (Thermo Fisher Scientific, Waltham, MA, USA) for sequencing using the Ion GeneStudio S5 Prime Sequencer (ThermoFisher Scientific, Waltham, MA, USA). Data were processed and analyzed through the Ion Reporter pipeline [17, 18].

\section{Reference Samples}

For initial platform validation, 2 HapMap samples (NA12877 and NA12878; Coriell Institute) and 2 reference DNA samples (TruQ2 and TruQ3; Horizon Discovery, UK) containing hot spot variants at $5 \%$ variant allele frequency (VAF) in GNAQ (p.Q209L) and GNA11 (p.Q209L) were tested using the Ion Ampliseq Cancer Hotspot v2 Library Kit (Thermo Fisher Scientific, Waltham, MA, 
Table 1. Genomic coordinates of 7-gene panel (reference sequence: hg19/grch37)

\begin{tabular}{llll}
\hline Gene & Transcript ID & $\begin{array}{l}\text { Genomic position } \\
\text { (start-stop) }\end{array}$ & $\begin{array}{l}\text { Region reported } \\
\text { (hotspot variant, if present) }\end{array}$ \\
\hline BAP1 & NM_004656.3 & chr3: 52436304-52443894 & All coding exons \pm 10 bp \\
CYSLTR2 & NM_020377.2 & chr13: 49281308-49281421 & Exon 1 (p.L129) \\
EIF1AX & NM_001412.3 & chrX: 20159723-20160042 & Exon 1 \pm 10 bp \\
EIF1AX & NM_001412.3 & chrX: 20156538-20156872 & Exon 2 \pm 10 bp \\
GNA11 & NM_002067.2 & chr19: 3118930-3119036 & Exon 5 (p.Q209) \\
GNA11 & NM_002067.2 & chr19: 3114958-3115053 & Exon 4 (p.R183) \\
GNAQ & NM_002072.3 & chr9: 80409443-80409558 & Exon 5 (p.Q209) \\
GNAQ & NM_002072.3 & chr9: 80412432-80412552 & Exon 4 (p.R183) \\
PLCB4 & NM_000933.3 & chr20: 9389729-9389853 & Exon 20 (p.D630) \\
SF3B1 & NM_012433.2 & chr2: 198267349-198267494 & Exon 14 (p.R625) \\
\hline
\end{tabular}

Fig. 1. Clinical workflow for the 7-gene panel. Primary tumor tissue (FFPE or FNAB) from a single biopsy is processed upon arrival at Castle Biosciences' clinical lab, with 1 aliquot used for RNA isolation and gene expression profiling and the other reserved for DNA isolation and sequencing. Targeted regions of the genome are amplified for sequencing on the Thermo Fisher Ion S5, after which variants are called using the Ion Reporter bioinformatics pipeline. Variants that meet minimum quality and coverage criteria and that are within reportable range are reported along with their potential clinical relevance according to the 4-tiered system for classification of somatic variants established by joint AMP, ASCO, and CAP guidelines [20]. AMP, Association for Molecular Pathology; CAP, College of American Pathologists; FFPE, formalinfixed paraffin-embedded; FNAB, fine-needle aspiration biopsy.

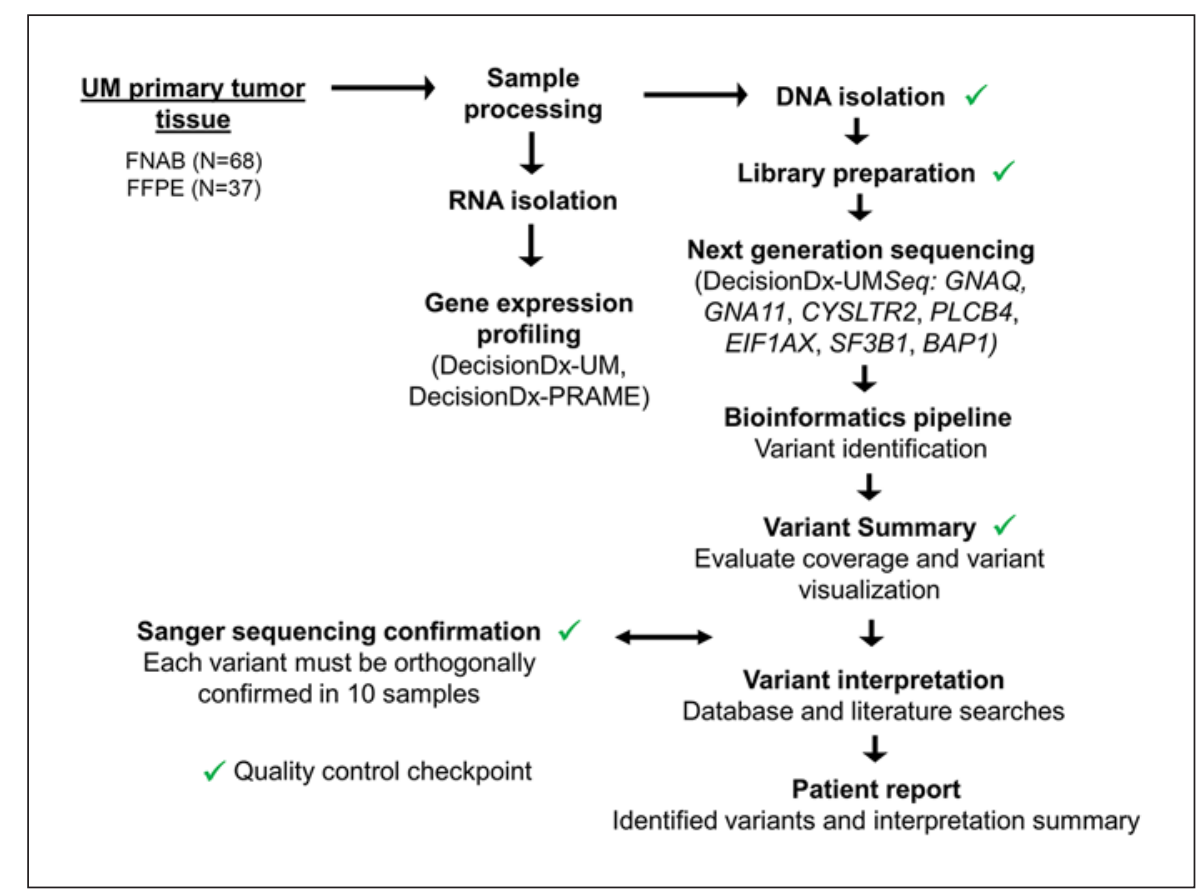

USA). To assess the lower limit of detection (LLOD), 6 FFPE samples and 13 FNAB samples with known VAFs were diluted with wild-type DNA (NA12878) as described previously [19].

\section{Orthogonal Confirmation of Sequence Variants}

Various orthogonal methods were used for this validation and are summarized in (online suppl. Table 1; for all online suppl. material, see www.karger.com/doi/10.1159/000518829). A subset of samples was sequenced on the Illumina MiSeq platform using a custom TruSeq panel and custom bioinformatics pipeline. A separate subset of samples underwent an inter-laboratory comparison with whole exome sequencing in 1 of 2 reference laboratories. The FNAB samples provided by the Harbour Lab were sequenced at the University of Miami Hussman Institute for Human Genomics (Miami, FL, USA), while the FFPE samples were sequenced at the Broad Institute (Cambridge, MA, USA). Furthermore, an addi- tional subset of samples was sent for Sanger sequencing - FNAB samples were sent to Elim Biopharmaceuticals (Hayward, CA, USA), and FFPE samples were sent to Eurofins Genomics (Louisville, KY, USA).

\section{Data Analysis}

Analysis was performed using Ion Torrent Suite Browser Version 5.8 and Ion Reporter Version 5.6 (reference sequence: hg19/ grch37). For each sequencing run, the ion sphere particle (ISP) density, yield, useable reads, and percent polyclonal were recorded to assess sequencing run quality metrics. Amplicon coverage, variant detection, and annotation were assessed for the regions of interest using Ion Reporter Version 5.6. Variants that met established quality control (QC) metrics were confirmed using orthogonal methods. Detection of deletions larger than $40 \mathrm{bp}$ was achieved by incorporating the following code into the ion reporter software: 
Table 2. Average quality and depth-of-coverage metrics across validation samples

\begin{tabular}{|c|c|c|c|c|c|c|c|c|}
\hline & \multicolumn{4}{|l|}{ Run metrics* } & \multicolumn{4}{|c|}{ Sample coverage ${ }^{* *}$} \\
\hline & $\begin{array}{l}\text { ISP density, } \\
\%\end{array}$ & $\begin{array}{l}\text { yield total } \\
\text { reads (M) }\end{array}$ & $\begin{array}{l}\text { useable } \\
\text { reads }\end{array}$ & $\begin{array}{l}\text { \% Poly- } \\
\text { clonal }\end{array}$ & $\begin{array}{l}\text { mapped } \\
\text { reads }\end{array}$ & $\begin{array}{l}\text { \% on } \\
\text { target }\end{array}$ & $\begin{array}{l}\text { mean } \\
\text { depth }\end{array}$ & $\begin{array}{l}\text { uniformity, } \\
\%\end{array}$ \\
\hline FNAB & 94 & 22.6 & $64 \%$ & 31 & 1,380,181 & 96 & 2,430 & 93 \\
\hline FFPE & 93 & 18.7 & $53 \%$ & 38 & $1,326,909$ & 96 & 2,288 & 93 \\
\hline Acceptable & $>70$ & $10-30$ & $>30 \%$ & $<50$ & $>200,000$ & $>80$ & $\geq 500 x$ & $\geq 80$ \\
\hline
\end{tabular}

FFPE, formalin-fixed paraffin-embedded; FNAB, fine-needle aspiration biopsy. ${ }^{*}$ Run metrics were averaged from 9 separate runs for FNAB samples and 8 separate runs for FFPE samples. ** Sample coverage data were averaged from the 68 FNAB samples and 37 FFPE samples used in the validation study.

tmap mapall .. -J 25 --end-repair 15 --bed-file --max-one-largeindel-rescue 40 --max-amplicon-overrun-large-indel-rescue 15 stage1 map4". As specified in the results section, variants that met the QC metrics were compared to sequencing results from other platforms in order to determine positive percent agreement (PPA) and technical positive predictive value (TPPV) for the target genes of the 7-gene panel.

\section{Results}

\section{Selection of Target Genes}

Genomic coordinates for the 7-gene panel were identified following literature and database searches for the regions of interest in order to define the reportable range $[15,20]$. The transcripts used for each gene along with the start and stop genomic positions for the targeted regions of interest are listed in Table 1. For clinical reporting purposes, all $B A P 1$ and $E I F 1 A X$ variants identified within 10 base pairs of the exons of interest are reported, while only known hotspot variants are reported for CYSLTR2, GNA11, GNAQ, PLCB4, and SF3B1. However, for purposes of this technical validation, all variants (including synonymous variants) within 10 base pairs of the exons of interest were reported for all genes, thereby including variants in exons and splice regions but excluding those in intronic regions.

\section{Sequencing Workflow}

To validate the 7-gene panel, a total of 68 FNAB and 37 FFPE samples were sequenced on the Thermo Fisher Ion S5. Following DNA extraction, enrichment of targeted genomic regions was performed by multiplex PCR using probes spanning the genes of interest. The sequencing workflow is outlined in Figure 1, with QC checkpoints indicated. Briefly, samples only passed QC if they achieved a minimum DNA concentration $>0.2 \mathrm{ng} / \mu \mathrm{L}$, and a library prep yield $>40 \mathrm{pM}$. Variants were reported if they achieved $>500 \mathrm{X}$ coverage per region of interest and a base quality score of $>100$ (Table 2).

\section{Sequencing Performance and Quality Control}

Table 2 summarizes the average quality and coverage metrics for both FNAB specimens $(N=68)$ and FFPE specimens $(N=37)$. The run metrics, including ISP density, total reads, percent of useable reads, and percent of polyclonal ISPs were all in line with the acceptable ranges recommended by the manufacturer.

Sample coverage data, including the mapped reads, percentage (\%) on target, mean depth of coverage, and uniformity were recorded. Mean depth of coverage was 2,430X for FNAB and 2,288X for FFPE, well above the $500 \mathrm{X}$ minimum recommended by the New York Clinical Laboratory Evaluation Program guidelines [21].

\section{Limits of Detection}

Serial dilution experiments determined that highquality sequencing results could be obtained with as little as $0.875 \mathrm{ng}$ of FNAB and $1.0 \mathrm{ng}$ of FFPE tissue (online suppl. Fig. 1). To assess the targeted LLOD of $5 \%$ VAF [19], 13 FNAB and 6 FFPE samples with known VAF were diluted to $5 \%$ with normal DNA. Sequencing of these samples resulted in correct detection of 26 of 27 variants (96\%) (online suppl. Table 2). To further confirm the assay's LLOD, sequencing was also performed on 2 well-characterized reference DNA samples, TruQ2, and TruQ3. These reference samples contain known, digitalPCR quantified mutations at low allele frequency (5\% $\mathrm{VAF}$ ) for 2 of the genes in the custom panel (GNAQ and GNA11). There was $100 \%$ concordance in identifying the target gene mutations in both reference DNA samples (data not shown), confirming that the sequencing platform accurately detects variants down to 5\% VAF. Fur- 
Table 3. Intra-assay concordance

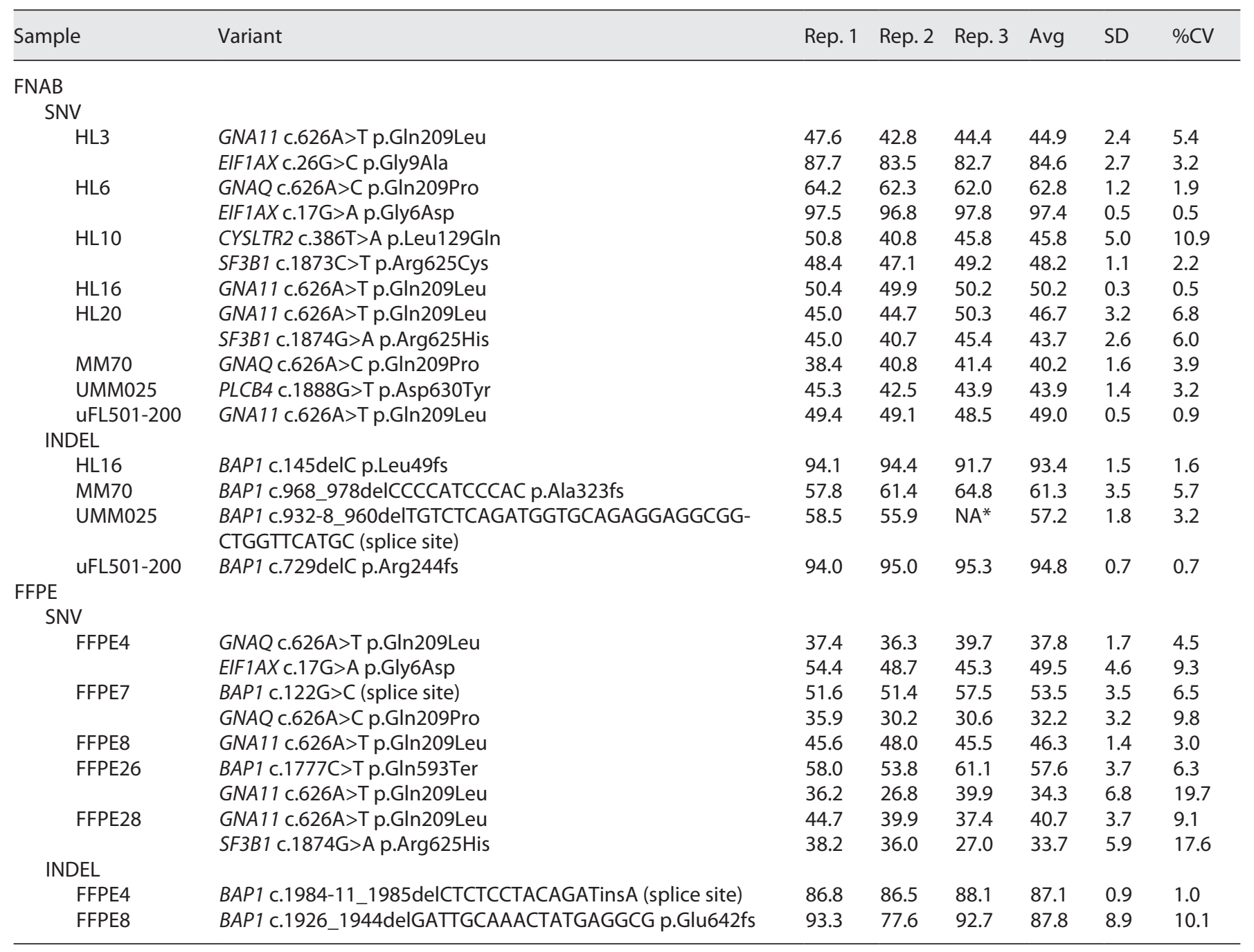

FFPE, formalin-fixed paraffin-embedded; FNAB, fine-needle aspiration biopsy; INDEL, insertions/deletions.

ther, sequencing of 2 HapMap DNA samples (NA12877 and NA12878) identified no reportable variants as expected.

\section{Precision}

Intra- and inter-assay concordance was evaluated by sequencing both FNAB and FFPE specimens in multiple replicates. To determine intra-assay concordance (Table 3), 8 unique FNAB samples and 5 unique FFPE samples were assayed in triplicate on the same chip with different barcodes. One-way ANOVA found no significant difference in the measured VAF for each variant detected (SNVs and in-frame deletions were analyzed separately). To determine inter-assay concordance (Table 4), 12 FNAB samples and 5 FFPE samples harboring SNVs in the 7 genes of interest were assayed on separate days across 4 different runs with different barcodes and by different operators. For INDEL analysis, 3 FNAB samples and 5 FFPE samples harboring BAP1 INDELs were assayed. Again, 1-way ANOVA determined no significant differences in the measured VAF between runs.

\section{Variant Identification in FNAB and FFPE Validation} Samples

A total of 129 variants (online suppl. Table 3) were identified across the 68 FNAB samples, and 69 variants (online suppl. Table 4) were identified across the 37 FFPE samples. Of note, $96 \%$ of samples harbored confirmed mutations in either GNA11 or GNAQ, $47 \%$ a BAP1 variant (either SNV or INDEL), 19\% a mutation in EIF1AX, 
Table 4. Inter-assay concordance

\begin{tabular}{|c|c|c|c|c|c|c|c|c|}
\hline Sample & Variant & Run 1 & Run 2 & Run 3 & Run 4 & Avg & SD & $\% C V$ \\
\hline \multicolumn{9}{|l|}{ FNAB } \\
\hline \multicolumn{9}{|l|}{ SNV } \\
\hline $\mathrm{HL} 2$ & GNA11 c.626A>T p.GIn209Leu & 46.7 & 52.2 & 48.5 & 50.3 & 49.4 & 2.37 & 4.8 \\
\hline \multirow[t]{2}{*}{ HL3 } & GNA11 c.626A>T p.GIn209Leu & 46.2 & 46.4 & 43.4 & 42.7 & 44.7 & 1.91 & 4.3 \\
\hline & EIF1AX c.26G >C p.Gly9Ala & 82.8 & 88.9 & 84.3 & 89.9 & 86.5 & 3.45 & 4.0 \\
\hline HL4 & GNA11 c.626A>T p.GIn209Leu & 48.0 & 46.0 & 43.4 & 48.9 & 46.6 & 2.45 & 5.3 \\
\hline \multirow[t]{2}{*}{ HL5 } & GNA11 c.626A>T p.GIn209Leu & 47.2 & 45.7 & 46.8 & 45.5 & 46.3 & 0.81 & 1.8 \\
\hline & EIF1AX c.17-2A >G (splice site) & 92.0 & 90.0 & 88.5 & 91.3 & 90.4 & 1.56 & 1.7 \\
\hline \multirow[t]{2}{*}{ HL6 } & GNAQ c.626A>C p.Gln209Pro & 66.9 & 64.7 & 66.7 & 65.8 & 66.0 & 1.00 & 1.5 \\
\hline & EIF1AX c.17G>A p.Gly6Asp & 96.9 & 97.2 & 95.6 & 98.1 & 97.0 & 1.03 & 1.1 \\
\hline HL7 & GNA11 c.626A>T p.GIn209Leu & 43.6 & 45.7 & 42.1 & 46.6 & 44.5 & 2.02 & 4.5 \\
\hline HL13 & GNAQ c.626A >C p.GIn209Pro & 62.0 & 61.1 & 60.4 & 61.3 & 61.2 & 0.66 & 1.1 \\
\hline \multirow[t]{2}{*}{ HL15 } & SF3B1 c. $1874 G>A$ p.Arg625His & 47.0 & 49.1 & 48.9 & 48.0 & 48.2 & 0.95 & 2.0 \\
\hline & GNAQ c.626A >T p.GIn209Leu & 42.9 & 45.9 & 47.9 & 46.5 & 45.8 & 2.11 & 4.6 \\
\hline \multirow[t]{2}{*}{ HL8 } & PLCB4 c. $1516 C>$ G p.GIn506Glu & 51.4 & 47.7 & 51.1 & 50.4 & 50.2 & 1.69 & 3.4 \\
\hline & GNA11 c.626A>T p.GIn209Leu & 39.2 & 40.2 & 37.3 & 39.2 & 39.0 & 1.21 & 3.1 \\
\hline HL16 & GNA11 c.626A>T p.GIn209Leu & 47.8 & 49.5 & 49.0 & 47.4 & 48.4 & 0.99 & 2.0 \\
\hline \multicolumn{9}{|c|}{ 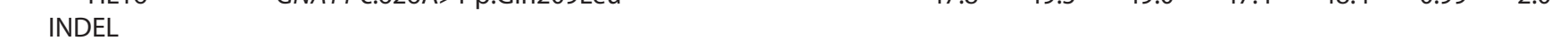 } \\
\hline HL7 & BAP1 c.898 899delAG p.Arq300fs & 78.4 & 74.0 & 77.2 & 76.2 & 76.5 & 1.86 & 2.4 \\
\hline HL12 & BAP1 c.853delT p.Ser285fs & 58.6 & 58.5 & 54.2 & 55.3 & 56.6 & 2.25 & 4.0 \\
\hline HL16 & BAP1 c.145delC p.Leu49fs & 92.8 & 93.1 & 92.7 & 92.5 & 92.8 & 0.25 & 0.3 \\
\hline \multicolumn{9}{|c|}{ 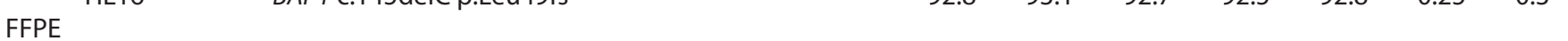 } \\
\hline \multicolumn{9}{|l|}{ SNV } \\
\hline FFPE32 & BAP1 c.1753_1774delTCCATCAGACCAA p.Ser585fs & 81.0 & 85.5 & 85.8 & 87.9 & 85.0 & 2.9 & 3.4 \\
\hline FFPE33 & BAP1 c.87_97delGGAAGGAGATCT & 93.7 & 95.6 & 95.0 & 94.8 & 94.8 & 0.8 & 0.8 \\
\hline FFPE35 & BAP1 c.1055_1056delCC p.Pro352fs & 64.6 & 74.7 & 76.1 & 71.5 & 71.7 & 5.1 & 7.2 \\
\hline
\end{tabular}

FFPE, formalin-fixed paraffin-embedded; FNAB, fine-needle aspiration biopsy; INDEL, insertions/deletions.

$16 \%$ a mutation in $S F 3 B 1,3 \%$ a mutation in $P L C B 4$, and $1 \%$ a mutation in CYSLTR2.

Because the purpose of this study was to validate the 7-gene panel for clinical use, only variants that would be clinically reported were included in the final analysis. Variant exclusions for the FNAB samples consisted of 1 outside of the reportable range (ORR) and 2 with low $(<15 \%)$ allele frequencies (AFs) that could not be confirmed with Sanger sequencing and were unable to be confirmed with additional orthogonal methods due to limited sample quantity. As the detection limit of Sanger sequencing is $15-20 \%$ [22], these variants were excluded from analysis. In the FFPE samples, variant exclusions consisted of 1 of 11 INDELs that was ORR, 2 SNVs that were ORR, and $1 \mathrm{SNV}$ with $\mathrm{AF}<15 \%$ which could not be confirmed by Sanger sequencing. Finally, 2 SNVs with AF $>15 \%$ were unable to be confirmed by Sanger sequencing and were thus deemed false positives. 


\section{Overall Performance Metrics}

After exclusions, a total of 126 variants from FNAB and 65 variants from FFPE samples were included in the comparison between the 7-gene panel results and the results from orthogonal sequencing methods (online suppl. Table 5). Overall in the combined validation cohort, there were 2 variants called by the Ion S5 that were not detected by orthogonal methods and were deemed false positives. There were no variants identified by orthogonal methods that were not also detected by the Ion S5 (false negatives).

For FNAB samples, 126 of 126 variants (SNV and INDEL) were correctly identified, resulting in an analytical PPA of $100 \%$ and TPPV of 100\%. For FFPE samples, 63 of 65 variants were correctly identified, with 2 false-positive results $(E I F 1 A X \mathrm{c} .17 \mathrm{G}>\mathrm{A}$ and $B A P 1 \mathrm{c} .67+2 \mathrm{~T}>\mathrm{G}$; online suppl. Table 5), resulting in a PPA of $100 \%$ and TPPV of $96.9 \%$. PPA and TPPV were also calculated based on variant type (SNV vs. INDEL) for the combined cohort of FNAB and FFPE samples. For detection of SNVs, the assay achieved $100 \%$ PPA and $98.8 \%$ TPPV, and for INDEL detection, it achieved 100\% PPA and 100\% TPPV. These results show that the 7-gene panel is robust, with consistent concordant results across multiple platforms with respect to variant identification.

\section{Discussion}

This study evaluated the analytical performance of a targeted NGS panel for mutational profiling of primary UM tumors. This 7-gene panel was validated for use in both FNAB and FFPE tissue from routine clinical samples across an array of parameters including sequencing performance, limit of detection, precision (reproducibility and repeatability), and measures of agreement (PPA and TPPV). It was also established that this panel can successfully be run from the same biopsy sample that is used for GEP testing. For samples in this validation that had both sequencing and GEP testing performed (31 FFPE and 64 FNAB), only 1 failed to yield a GEP result, resulting in a technical success rate of $98.9 \%$.

Consistent with prior studies evaluating mutation frequencies in UM tumors [7, 11-15, 23-28], 96\% of samples analyzed in this validation harbored mutually exclusive mutations in either GNA11 or GNAQ. Similarly, 47\% of samples harbored a $B A P 1$ variant (either SNV or INDEL). Mutations in EIF1AX, SF3B1, PLCB4, and CYSLTR2 were less frequent, occurring in $20 \%, 16 \%, 3 \%$, and $1 \%$ of tumors, respectively. Of interest, 1 FNAB sample harbored concurrent mutations in GNA11 and PLCB4, which have been reported to occur in a mutually exclusive manner $[9,14]$.

Inclusion of probes tiled across the entire $B A P 1$ gene locus allowed for the identification of 22 novel BAP1 variants that were previously unreported in Clinvar, COSMIC, Leiden Open Variation Database, and HGMD Professional release 2020.2 [29-32]. These novel BAP1 variants include $1 \mathrm{SNV}$ (c.254A>T) and 20 INDELs (online suppl. Tables 3,4 ), most of which were small, with only 1 over $50 \mathrm{bp}$. One of these novel BAP1 deletions (c.729delC) was identified in 2 primary tumors that were biopsied from the same patient who experienced growth of a second primary tumor 2 years after initial intervention, consistent with intraocular metastasis [33]. All of these novel $B A P 1$ variants were orthogonally validated by Sanger sequencing. Thus, we demonstrate the capabilities of this platform to capture potentially clinically significant mutations that allow for risk stratification and serve as entry criteria in BAP1 targeted therapy clinical trials.

The present study was designed to assess and validate the processes, methods, and performance of the 7-gene sequencing panel. While rigorous analytic validation is essential for establishing the performance characteristics of any clinically available laboratory developed test, we recognize that this study is limited in that it did not examine the correlation between tumor mutations and GEP results, PRAME results, and/or clinical outcomes. However, a large, multicenter prospective clinical trial (the Collaborative Ocular Oncology Group Uveal Melanoma Validation Study Number 2 or COOG2) is currently underway to evaluate the relationship between GEP, PRAME, and common driver mutations in UM using this validated 7-gene panel.

\section{Conclusion}

The 7-gene panel is a robust, highly accurate NGS test that is unique compared to other sequencing tests in that it can be performed from the same biopsy sample that is used for GEP and does not require a second biopsy. The ability to perform mutational profiling of primary UM tumors in addition to GEP is important as it may aid in confirming the presence of a melanocytic lesion, potentially better inform therapy selection, and provide additional layers of prognostic information to help guide patient management decisions. 


\section{Statement of Ethics}

Institutional Review Board approval was not required for the existing residual clinical specimens at Castle Biosciences because this analysis is exempt from the regulatory review requirements as set forth in section 46.101 (b) of 45 CFR 46. De-identified specimens provided by the Harbour Lab were obtained with written patient informed consent and approval of the Institutional Review Board of the University of Miami (IRB-approved protocol number 20120773).

\section{Conflict of Interest Statement}

K.M.A., L.M.S., K.R.C., S.M.A., J.K.W., J.F.S., S.B., K.M.O., F.A.M., and R.W.C. were employees and stockholders of Castle Biosciences, Inc. at the time of this study. J.W.H. is the inventor of intellectual property related to prognostic testing for uveal melanoma. He is a paid consultant for Castle Biosciences, Inc., Friendswood, TX, USA licensee of this intellectual property, and he receives royalties from its commercialization.

\section{Funding Sources}

This work was supported by Castle Biosciences, Inc., Friendswood, TX, USA and by National Cancer Institute grant R01 CA125970 (J.W.H.), Melanoma Research Foundation Established Investigator Award (J.W.H.), the University of Miami Miller
School of Medicine Medical Scientist Training Program (M.A.D.), the University of Miami Sheila and David Fuente Graduate Program in Cancer Biology (M.A.D.), the Center for Computational Science Fellowship (M.A.D.), and a generous gift from Dr. Mark J. Daily (J.W.H.). The Bascom Palmer Eye Institute also received funding from NIH Core Grant P30 EY014801 and a Research to Prevent Blindness Unrestricted Grant. The Sylvester Comprehensive Cancer Center also received funding from the National Cancer Institute of the National Institutes of Health under Award Number P30CA240139. The content is solely the responsibility of the authors and does not necessarily represent the official views of the National Institutes of Health.

\section{Author Contributions}

K.M.A., L.M.S., and S.B. had full access to the data in this study and take responsibility for its integrity. All other co-authors contributed to study design, data analysis/interpretation, and critical review of the manuscript prior to submission.

\section{Data Availability Statement}

All data generated or analyzed during this study are included in this article [and/or] its online suppl. material files. A preliminary version of this article was made available on a preprint server with DOI 10.21203/rs.3.rs-86927/v1. Further inquiries can be directed to the corresponding author.

\section{References}

1 Kujala E, Mäkitie T, Kivelä T. Very long-term prognosis of patients with malignant uveal melanoma. Invest Ophthalmol Vis Sci. 2003 Nov 1;44(11):4651.

2 Onken MD, Worley LA, Char DH, Augsburger JJ, Correa ZM, Nudleman E, et al. Collaborative ocular oncology group report number 1: prospective validation of a multi-gene prognostic assay in uveal melanoma. Ophthalmology. 2012 Aug;119(8):1596-603.

3 Aaberg TM, Covington KR, Tsai T, Shildkrot Y, Plasseraud KM, Alsina KM, et al. Gene expression profiling in uveal melanoma: fiveyear prospective outcomes and meta-analysis. Ocul Oncol Pathol. 2020 Jul 6;6:360-7.

4 Plasseraud KM, Wilkinson JK, Oelschlager KM, Poteet TM, Cook RW, Stone JF, et al. Gene expression profiling in uveal melanoma: technical reliability and correlation of molecular class with pathologic characteristics. Diagn Pathol. 2017 Dec;12(1):59.

5 National Comprehensive Cancer Network (NCCN). NCCN Clinical Practice Guidelines in Oncology. Uveal Melanoma Version 2. 2021 National Comprehensive Cancer Network. Available from: https://www.nccn.org/ guidelines/guidelines-detail? category $=1 \&$ id $=1488$.
6 Amin MB, Edge MB, Greene FL, Byrd DR, Brookland RK, Washington ML, et al. American Joint Committee on Cancer (AJCC) staging manual, Vol. 8. New York: Springer; 2017.

7 Decatur CL, Ong E, Garg N, Anbunathan H, Bowcock AM, Field MG, et al. Driver mutations in uveal melanoma: associations with gene expression profile and patient outcomes. JAMA Ophthalmol. 2016 Jul 1;134(7):728-33.

8 Johansson P, Aoude LG, Wadt K, Glasson WJ, Warrier SK, Hewitt AW, et al. Deep sequencing of uveal melanoma identifies a recurrent mutation in PLCB4. Oncotarget. 2015 Dec 14; 7(4):4624-31.

9 Moore AR, Ceraudo E, Sher JJ, Guan Y, Shoushtari AN, Chang MT, et al. Recurrent activating mutations of G-protein-coupled receptor CYSLTR2 in uveal melanoma. Nat Genet. 2016 Jun;48(6):675-80.

10 Field MG, Durante MA, Anbunathan H, Cai LZ, Decatur CL, Bowcock AM, et al. Punctuated evolution of canonical genomic aberrations in uveal melanoma. Nat Commun. 2018 Jan 9 [cited 2020 Mar 31];9:116.

11 Onken MD, Worley LA, Long MD, Duan S, Council ML, Bowcock AM, et al. Oncogenic mutations in GNAQ occur early in uveal melanoma. Invest Ophthalmol Vis Sci. 2008 Dec; 49(12):5230-4.
12 Van Raamsdonk CD, Bezrookove V, Green G, Bauer J, Gaugler L, O'Brien JM, et al. Frequent somatic mutations of GNAQ in uveal melanoma and blue naevi. Nature. 2009 Jan 29;457(7229):599-602.

13 Van Raamsdonk CD, Griewank KG, Crosby MB, Garrido MC, Vemula S, Wiesner T, et al. Mutations in GNA11 in uveal melanoma. N Engl J Med. 2010 Dec 2;363(23):2191-9.

14 Robertson AG, Shih J, Yau C, Gibb EA, Oba $\mathrm{J}$, Mungall KL, et al. Integrative analysis identifies four molecular and clinical subsets in uveal melanoma. Cancer Cell. 2017 Aug 14; 32(2):204-20.e15.

15 Harbour JW, Onken MD, Roberson ED, Duan S, Cao L, Worley LA, et al. Frequent mutation of BAP1 in metastasizing uveal melanomas. Science. 2010 Dec 3;330(6009): $1410-3$.

16 U.S. Food and Drug Administration. Informed consent for in vitro diagnostic device Studies using leftover human specimens that are not individually identifiable [Internet]. 2006. Available from: https://www.fda.gov/ media/122648/download.

17 MAN0017530 Torrent Suite Software 5.8 User Guide 01.09.2018. 
18 Roy S, Coldren C, Karunamurthy A, Kip NS, Klee EW, Lincoln SE, et al. Standards and guidelines for validating next-generation sequencing bioinformatics pipelines: a joint recommendation of the association for molecular pathology and College of American Pathologists. J Mol Diagn. 2018 Jan;20(1):427.

19 Jennings LJ, Arcila ME, Corless C, Kamel-Reid S, Lubin IM, Pfeifer J, et al. Guidelines for validation of next-generation sequencingbased oncology panels: a joint consensus recommendation of the association for molecular pathology and College of American $\mathrm{Pa}$ thologists. J Mol Diagn. 2017 Mar 20;19: 341-65.

20 Li MM, Datto M, Duncavage EJ, Kulkarni S, Lindeman NI, Roy S, et al. Standards and guidelines for the interpretation and reporting of sequence variants in cancer: a joint consensus recommendation of the association for molecular pathology, American Society of Clinical Oncology, and College of American Pathologists. J Mol Diagn. 2017 Jan;19(1):423.

21 NYS DOH Wadsworth Center. Clinical laboratory evaluation program guide [Internet]. 2020. Available from: https://www.wadsworth.org/sites/default/files/WebDoc/ CLEP_program-guide_july2020.pdf.

22 Tsiatis AC, Norris-Kirby A, Rich RG, Hafez MJ, Gocke CD, Eshleman JR, et al. Compari- son of Sanger sequencing, pyrosequencing, and melting curve analysis for the detection of KRAS mutations: diagnostic and clinical implications. J Mol Diagn. 2010 Jul;12(4):42532.

23 Bauer J, Kilic E, Vaarwater J, Bastian BC, Garbe C, de Klein A. Oncogenic GNAQ mutations are not correlated with disease-free survival in uveal melanoma. Br J Cancer. 2009 Sep 1;101(5):813-5.

24 Dono M, Angelini G, Cecconi M, Amaro A, Esposito AI, Mirisola V, et al. Mutation frequencies of GNAQ, GNA11, BAP1, SF3B1, EIF1AX and TERT in uveal melanoma: detection of an activating mutation in the TERT gene promoter in a single case of uveal melanoma. Br J Cancer. 2014 Feb 18;110(4):105865.

25 Martin M, Maßhöfer L, Temming P, Rahmann S, Metz C, Bornfeld N, et al. Exome sequencing identifies recurrent somatic mutations in EIF1AX and SF3B1 in uveal melanoma with disomy 3. Nat Genet. 2013 Aug; 45(8):933-6.

26 Harbour JW, Roberson ED, Anbunathan $\mathrm{H}$, Onken MD, Worley LA, Bowcock AM. Recurrent mutations at codon 625 of the splicing factor SF3B1 in uveal melanoma. Nat Genet. 2013 Feb;45(2):133-5.

27 Afshar AR, Damato BE, Stewart JM, Zablotska LB, Roy R, Olshen AB, et al. Next-generation sequencing of uveal melanoma for detec- tion of genetic alterations predicting metastasis. Trans Vis Sci Tech. 2019 Apr 17;8(2): 18.

28 Thornton S, Coupland SE, Olohan L, Sibbring JS, Kenny JG, Hertz-Fowler C, et al. Targeted next-generation sequencing of 117 routine clinical samples provides further insights into the molecular landscape of uveal melanoma. Cancers. 2020 Apr;12(4):1039.

29 Stenson PD, Ball EV, Mort M, Phillips AD, Shiel JA, Thomas NS, et al. Human gene mutation database (HGMD): 2003 update. Hum Mutat. 2003 Jun;21(6):577-81.

30 Fokkema IF, Taschner PE, Schaafsma GC, Celli J, Laros JF, den Dunnen JT. LOVD v.2.0: the next generation in gene variant databases. Hum Mutat. 2011 May;32(5):557-63.

31 Landrum MJ, Lee JM, Benson M, Brown GR, Chao C, Chitipiralla S, et al. ClinVar: improving access to variant interpretations and supporting evidence. Nucleic Acids Res. 2018 Jan 4;46(D1):D1062-7.

32 Tate JG, Bamford S, Jubb HC, Sondka Z, Beare DM, Bindal N, et al. COSMIC: the catalogue of somatic mutations in cancer. Nucleic Acids Res. 2019 Jan 8;47(D1):D941-7.

33 Durante MA, Walter SD, Paez-Escamilla M, Tokarev J, Decatur CL, Dubovy SR, et al. Intraocular metastasis in unilateral multifocal uveal melanoma without melanocytosis or germline BAP1 mutations. JAMA Ophthalmol. 2019 Dec;137(12):1434-9. 